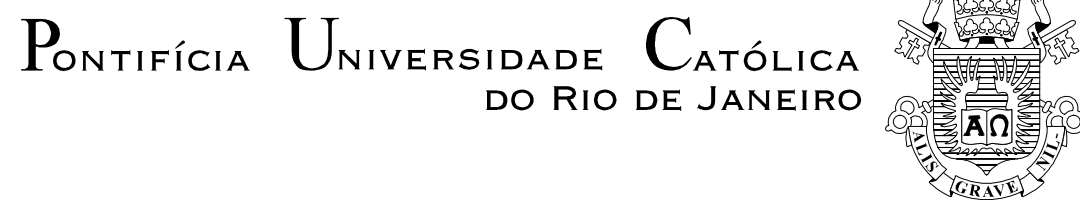

Leonardo Mendes Simão

Otimização da Programação da Produção em Refinarias de Petróleo utilizando Algoritmos Genéticos e Co-evolução Cooperativa.

Dissertação de Mestrado

Dissertação apresentada como requisito parcial para obtenção do título de Mestre pelo Programa de Pós-Graduação em Engenharia Elétrica da PUC-Rio.

Orientador: Marco Aurélio C. Pacheco Co-orientador: Carlos R. Hall Barbosa 


$$
\begin{array}{r}
\text { Pontifícia Universidade Católica } \\
\text { do Rio de Janeiro }
\end{array}
$$

Leonardo Mendes Simão

\section{Otimização da Programação da Produção em Refinarias de Petróleo Utilizando Algoritmos Genéticos e Co-Evolução Cooperativa}

Dissertação de Mestrado apresentada como requisito parcial para obtenção do grau de Mestre pelo Programa de PósGraduação em Engenharia Elétrica do Departamento de Engenharia Elétrica do Centro Técnico Científico da PUC-Rio. Aprovada pela Comissão Examinadora abaixo assinada.

Prof. Marco Aurélio Cavalcanti Pacheco Orientador Departamento de Engenharia Elétrica - PUC-Rio

Prof. Carlos Roberto Hall Barbosa Co-Orientador Departamento de Engenharia Elétrica - PUC-Rio

Prof. Sergio Augusto Barreto da Fontoura Departamento de Engenharia Civil - PUC-Rio

Prof. Silvio Hamacher Departamento de Engenharia Industrial - PUC-Rio

Prof. Paulo Leo Manassi Osório Departamento de Engenharia Elétrica - PUC-Rio

Prof. José Eugenio Leal Coordenador Setorial do Centro Técnico Científico - PUC-Rio 
Todos os direitos reservados. É proibida a reprodução total ou parcial do trabalho sem autorização da universidade, do autor e do orientador.

Leonardo Mendes Simão

Graduado em Engenharia de Computação pela Pontifícia Universidade Católica do Rio de Janeiro (PUC-Rio) em 1999.

Ficha Catalográfica

Simão, Leonardo Mendes

Otimização da programação da produção em refinarias de petróleo utilizando algoritmos genéticos e co-evolução cooperativa / Leonardo Mendes Simão ; orientador: Marco Aurélio C. Pacheco ; co-orientador: Carlos R. Hall Barbosa. - Rio de Janeiro : PUC, Departamento de Engenharia Elétrica, 2004.

103 f. : il. ; $30 \mathrm{~cm}$

Dissertação (mestrado) - Pontifícia Universidade Católica do Rio de Janeiro, Departamento de Engenharia Elétrica.

Inclui referências bibliográficas.

1. Engenharia elétrica - Teses. 2. Computação evolucionária. 3. Algoritmos genéticos. 4. Co-evolução cooperativa. 5. Programação da produção. 6. Refinaria. 7. Petróleo. 8. Indústria. I. Pacheco, Marco Aurélio C. II. Barbosa, Carlos R. Hall. III. Pontifícia Universidade Católica do Rio de Janeiro. Departamento de Engenharia Elétrica. IV. Título. 


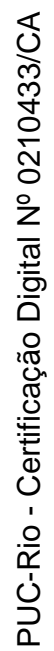

Para Vanessa 


\section{Agradecimentos}

Ao meu orientador, Prof. Dr. Marco Aurélio C. Pacheco, pela orientação, apoio e estímulo, nesta empreitada. E ao meu co-orientador, Prof. Dr. Carlos R. Hall Barbosa, pelas inestimáveis contribuições à pesquisa desenvolvida.

Ao colega André Vargas Cruz pelo conhecimento compartilhado e pelo tempo dedicado.

Aos colegas da PUC-Rio: Cristiane Camacho, Flávia Lessa, Rafael Olivaes, Dan Posternak, Karla Figueiredo e Luciana Faletti.

Ao amigo Fabio Fagundez pela parceria nos momentos difíceis da jornada. E aos colegas da Chemtech: Paulo Armando, Rodrigo Lopes, Alessandro Alle, Cristiane Fukasawa, Bruno Queiroz, Franklin Walter, Bianca Oliveira, Laurinei Pimentel e Rüdiger Reichow. Tenho orgulho de fazer parte desta equipe.

Ao amigo Marcos Vinícius Gusmão Nascimento, pelo apoio e incentivo.

Aos meus pais, pelo apoio, educação e por ser quem são.

Aos meus amigos e familiares pela compreensão na minha ausência.

À minha esposa, Vanessa, pelo carinho, apoio incondicional e por existir em minha vida.

À minha filha, Marina, que apesar de não ter nascido ainda, já me incentiva a ser uma pessoa melhor. 


\section{Resumo}

Simão, Leonardo Mendes. Otimização da Programação da Produção em Refinarias de Petróleo utilizando Algoritmos Genéticos e Co-evolução Cooperativa. Pontifícia Universidade Católica do Rio de Janeiro, 2004. 103p. Dissertação de Mestrado - Departamento de Engenharia Elétrica, Pontifícia Universidade Católica do Rio de Janeiro.

Esta dissertação investiga a aplicação de Algoritmos Genéticos e de CoEvolução Cooperativa na otimização da programação da produção em refinarias de petróleo. Refinarias de petróleo constituem um dos mais importantes exemplos de plantas contínuas multiproduto, isto é, um sistema de processamento contínuo gerador de múltiplos produtos simultâneos. Uma refinaria, em geral, processa um ou mais tipos de petróleo, produzindo uma série de produtos derivados, como o GLP (gás liquefeito de petróleo), a nafta, o querosene e o óleo diesel. Trata-se de um problema complexo de otimização, devido ao número e diversidade de atividades existentes e diferentes objetivos. Além disso, neste problema, algumas atividades dependem de que outras atividades já tenham sido planejadas para que possam então ser planejadas. Um caso típico é o das retiradas de produtos de uma unidade de processo, que dependem de que a carga já tenha sido planejada, assim como em qual campanha a unidade estará naquele instante. Por isso, o uso de modelos evolucionários convencionais, como os baseados em ordem, pode gerar muitas soluções inválidas, que deverão ser posteriormente corrigidas ou descartadas, comprometendo o desempenho e a viabilidade do algoritmo. O objetivo do trabalho foi, então, desenvolver um modelo evolucionário para otimizar a programação da produção (scheduling), segundo objetivos bem definidos, capaz de lidar com as restrições do problema, gerando apenas soluções viáveis.

O trabalho consistiu em três etapas principais: um estudo sobre o refino de petróleo e a programação da produção em refinarias; a definição de um modelo usando algoritmos genéticos e co-evolução cooperativa para otimização da 
programação da produção e a implementação de uma ferramenta para estudo de caso.

O estudo sobre o refino e a programação da produção envolveu o levantamento das várias etapas do processamento do petróleo em uma refinaria, desde o seu recebimento, destilação e transformação em diversos produtos acabados, que são então enviados a seus respectivos destinos. Neste estudo, também foi levantada a estrutura de tomada de decisão em uma refinaria e seus vários níveis, diferenciando os objetivos destes níveis e explicitando o papel da programação da produção nesta estrutura. A partir daí, foram estudadas em detalhes todas as atividades que normalmente ocorrem na refinaria e que são definidas na programação, e seus papéis na produção da refinaria. A decisão de quando e com que recursos executar estas atividades é o resultado final da programação e, portanto, a saída principal do algoritmo.

A modelagem do algoritmo genético consistiu inicialmente em um estudo de representações utilizadas para problemas de scheduling. O modelo coevolucionário adotado considera a decomposição do problema em duas partes e, portanto, emprega duas populações com responsabilidades diferentes: uma é responsável por indicar quando uma atividade deve ser planejada e a outra é responsável por indicar com quais recursos essa mesma atividade deve ser realizada. A primeira população teve sua representação baseada em um modelo usado para problemas do tipo "Dial-A-Ride” (Moon et al, 2002), que utiliza um grafo para indicar à função de avaliação a ordem na qual o planejamento deve ser construído. Esta representação foi elaborada desta forma para que fosse levada em conta a existência de restrições de precedência (atividades que devem ser planejadas antes de outras), e assim não fossem geradas soluções inválidas pelo algoritmo. A segunda população, que se responsabiliza pela alocação dos recursos para a execução das atividades, conta com uma representação onde os operadores genéticos podem atuar na ordem de escolha dos recursos que podem realizar cada uma das atividades.

Finalmente, desenvolveu-se uma ferramenta para implementar estes modelos e tratar de um estudo de caso, que oferecesse as características necessárias para testar a qualidade das representações e avaliar os resultados. Foi criada uma refinaria bem simples, mas que contasse com todos os tipos de 
equipamentos, atividades e restrições presentes em uma refinaria real. As restrições existentes são tanto as de precedência, que são incorporadas através do grafo utilizado pela primeira espécie, quanto às restrições operacionais inerentes ao problema e à planta escolhida. Com isso, foi possível validar o processo de decodificação dos cromossomos em soluções viáveis, respeitando as restrições do problema.

Foram realizados vários testes que demonstraram a capacidade dos modelos desenvolvidos em gerar soluções viáveis, sem a necessidade de heurísticas de correção, e os resultados obtidos foram comparados com os de um processo de busca exaustiva.

Foram criados três cenários de teste com demandas, restrições e tamanhos diferentes. Em todos os casos, os resultados obtidos pelos modelos foram sempre muito superiores aos da busca exaustiva.

\section{Palavras-chave}

Computação Evolucionária; Algoritmos Genéticos; Co-evolução Cooperativa; Programação da Produção; Refinaria; Petróleo; Indústria. 


\section{Abstract}

Simão, Leonardo Mendes. Refinery Scheduling Optimization using Genetic Algorithms and Cooperative Coevolution. Pontifícia Universidade Católica do Rio de Janeiro, 2004. 103p. Dissertação de Mestrado - Departamento de Engenharia Elétrica, Pontifícia Universidade Católica do Rio de Janeiro.

This work investigates the use of Genetic Algorithms and Cooperative Coevolution in refinery scheduling optimization. Oil refineries are one of the most important examples of multiproduct continuous plants, that is, a continuous processing system that generates a number of products simultaneously. A refinery processes various crude oil types and produces a wide range of products, including LPG (liquefied petroleum gas), gasoline, kerosene and diesel. It is a complex optimization problem, mainly due to the number of different tasks involved and different objective criteria. In addition, some of the tasks have precedence constraints that require other tasks to be scheduled first. For example, in order to schedule a task that transfers one of the yields of a certain crude distillation unit, both the task that feeds the crude oil into the unit and the task that sets the unit's current operation mode must already be scheduled. Therefore, applying traditional evolutionary models, like the order-based ones, can create many infeasible solutions that will have to be corrected or rejected later on, thereby jeopardizing the algorithm performance and feasibility. The main goal was the development an evolutionary model satisfying well-defined objectives, which would optimize production scheduling and address the various constraints entailed in the problem, thus generating only feasible solutions.

This work consisted on three main steps: a survey on crude oil refining and refinery scheduling; the development of a cooperative coevolutionary model to optimize the refinery scheduling and the development of a software tool for case studies.

The study about refining and scheduling involved gathering information 
about the existent processes in a refinery, starting from the arrival of crude oil, its distillation and transformation into several products and, finally, the delivery of these products to their respective destination. The levels of decision making in a refinery were surveyed too, in order to identify the main goals for each one, and how the scheduling level fits into the structure as whole. Then, all the routine scheduling tasks and their roles in a refinery were carefully studied. The decision of when and how to assign those tasks is the final output of the scheduling task, so it must be the main output of the algorithm too.

The development of the evolutionary model consisted of a survey on some of the most common evolutionary approaches to scheduling. The adopted coevolutionary model breaks the problem down into two parts, thus using two species with different responsibilities: One is responsible for deciding when a task should be scheduled, while the other is responsible for assigning a resource for this task. The first species representation was based on a model used for the “Dial-a-Ride” (Moon et al, 2002) kind of problems, and uses a graph to help the fitness evaluation function find the right order in which to schedule the tasks. This representation was devised in such a way that the precedence constraints were satisfied and no infeasible solutions were generated. The representation of the second species, which assigns resources for the tasks, let genetic operators change the selection order when picking a resource for a task.

Finally, a software tool was developed to be used for implement this model and for performing a case study. This case study should comprise all the needed characteristics, in order to test the quality of the representation as well as evaluate the results. A simple refinery was designed, containing all equipment types, tasks and constraints found in a real-world refinery. The constraints mentioned are the precedence constraints, handled by the graph used by the first species, plus other operational constraints found in refinery scheduling. It was possible, then, to see the decoding of chromosomes into feasible solutions, always satisfying all the constraints.

Several tests were performed, and they showed that the model developed is, in fact, capable of generating and maintaining feasible solutions, without repairing, penalizing or discarding them. The results were compared to the ones obtained through exhaustive search. 
Three test scenarios were designed with different sizes, demands and constraints. In all of them, the results obtained were far better than the ones obtained through exhaustive search.

\section{Keywords}

Evolutionary Computation; Genetic Algorithms; Cooperative Coevolution; Scheduling; Refinery; Production; Industry. 


\section{Sumário}

1 . Introdução. 19

1.1. Motivação 19

1.2. Objetivo 20

1.3. Descrição do Trabalho 21

1.4. Conteúdo da Dissertação 23

2. Descrição do Problema. 24

2.1. O Refino de Petróleo $\quad 24$

2.2. Planejamento da Produção (Planning). 25

2.3. A Programação da Produção. 26

2.3.1. Processos envolvidos. 26

2.3.2. O Problema da Programação da Produção. 33

3 . Algoritmos Genéticos e Co-evolução Cooperativa. 36

3.1. Introdução. 36

3.2. Representação de Problemas de Scheduling. 40

3.2.1. Abordagens Anteriores do Problema utilizando Algoritmos

Genéticos. 43

3.2.2. Alternativas à computação evolucionária 48

3.3. Co-evolução. 49

3.3.1. Co-evolução Cooperativa. 51

4 Modelo Co-evolucionário para Otimização da Programação da Produção.

4.1. Introdução.

4.2. Representação. 56

4.3. Decodificação. 61

4.4. Avaliação. 62

4.5. Operadores. 64

4.6. Representação do Tempo. 64 
5. Estudo de Caso. 67

5.1. Descrição do Protótipo. $\quad 67$

5.2. Grafo de Precedências. 68

5.3. Cenários estudados $\quad 69$

5.4. Resultados 73

5.4.1. Análise de Desempenho. 73

5.4.2. Programação Gerada pelo Decodificador. 84

6 Conclusões e Trabalhos Futuros. 97

7 . Referências. 100 


\section{Lista de Figuras}

Figura 1-Esquema do Processo de Refino............................................................ 25

Figura 2-Esquema Simplificado de uma Refinaria de Petróleo. ........................... 27

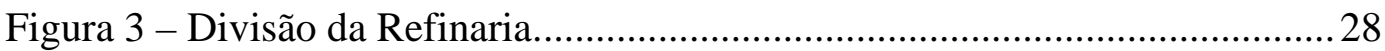

Figura 4-Fluxo básico de um algoritmo evolucionário. ........................................ 37

Figura 5-Arquitetura do Modelo Co-evolucionário. ........................................... 52

Figura 7 - Algoritmo para programar tarefas a partir da espécie 1....................... 58

Figura 8 - Passo 1: Seleciona a atividade A (única sem predecessores)................ 58

Figura 9 - Passo 2: Seleciona a atividade B (entre B e C, B vem primeiro na

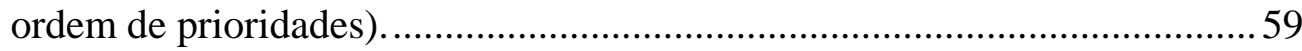

Figura 10 - Passo 3: Seleciona a atividade C (entre D e C, C vem primeiro na ordem de prioridades).

59

Figura 11 - Passo 4: Seleciona a atividade E (entre D e E, E vem primeiro na ordem de prioridades).

Figura 12 - Passo 5: Seleciona a atividade E (única de predecessor), e assim por diante. 60

Figura 13 - Representação da espécie “Alocação de Recurso”. ............................ 60

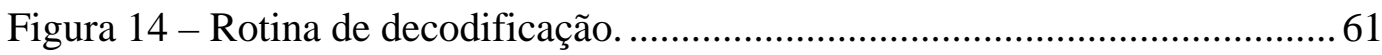

Figura 15 - Refinaria do Protótipo......................................................................67

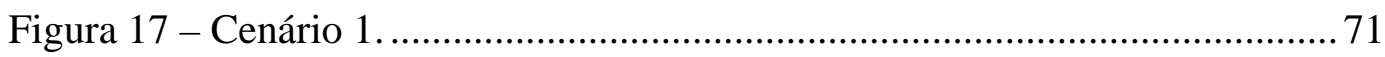

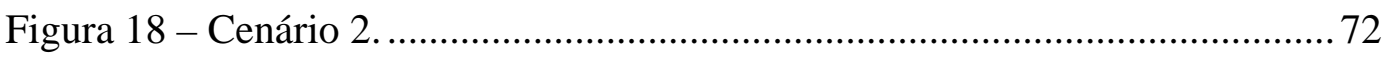

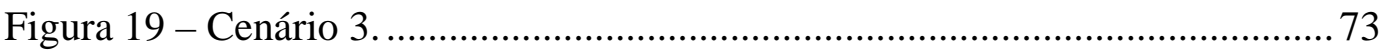

Figura 20 - Desempenho do Algoritmo em relação à busca exaustiva (Cenário 1). 74

Figura 21 - Custo por Atraso nos Itens - Algoritmo X Busca exaustiva (Cenário 1). .74

Figura 22 - Custo de Matéria-Prima - Algoritmo X Busca exaustiva (Cenário 1). .75

Figura 23 - Trocas de Tanques - Algoritmo X Busca exaustiva (Cenário 1)....... 75 Figura 24 - Custo por Desvio de Especificação - Algoritmo X Busca (Cenário 1). 
Figura 25 - Avaliação dos operadores (Cenário 1).

Figura 26 - Desempenho do Algoritmo em relação à busca exaustiva (Cenário 2). 78

Figura 27 - Custo por Atraso nos Itens - Algoritmo X Busca exaustiva (Cenário 2). 79

Figura 28 - Custo de Matéria-Prima - Algoritmo X Busca exaustiva (Cenário 2). .79

Figura 29 - Trocas de Tanques - Algoritmo X Busca exaustiva (Cenário 2)....... 79 Figura 30 - Custo por Desvio de Especificação - Algoritmo X Busca (Cenário 2). 80

Figura 31 - Desempenho do Algoritmo em relação à busca exaustiva (Cenário 3). .81

Figura 32 - Custo por Atraso nos Itens - Algoritmo X Busca exaustiva (Cenário 3). 81

Figura 33 - Custo de Matéria-Prima - Algoritmo X Busca exaustiva (Cenário 3).

Figura 34 - Trocas de Tanques - Algoritmo X Busca exaustiva (Cenário 3)....... 82 Figura 35 - Custo por Desvio de Especificação - Algoritmo X Busca (Cenário 3).

Figura 36 - Trecho de ordens de produção........................................................... 84

Figura 37 - Gráfico de Gantt com toda a programação (Cenário 1)...................... 85

Figura 38 - Gráfico de Volumes nos Tanques de Cru (Cenário 1)........................87

Figura 39 - Gráfico de Volumes nos Tanques de Nafta (Cenário 1). ................... 87

Figura 40 - Gráfico de Volumes nos Tanques de Querosene (Cenário 1)............ 88

Figura 41 - Gráfico de Volumes nos Tanques de Diesel (Cenário 1)................... 88

Figura 42 - Gráfico de Volumes nos Tanques de Gasóleo e Diluente (Cenário 1). 89

Figura 43 - Gráfico de Volumes nas Esferas de GLP (Cenário 1)........................89

Figura 44 - Gráfico de densidade nos tanques de diesel (Cenário 1).................... 90

Figura 45 - Gráfico de enxofre nos tanques de diesel (Cenário 1)...................... 90

Figura 46 - Gráfico de Gantt com toda a programação (Cenário 2)..................... 91

Figura 47 - Gráfico de Volumes nos Tanques de Cru (Cenário 2)...................... 93

Figura 48 - Gráfico de Volumes nos Tanques de Nafta (Cenário 2). ................... 93 
Figura 49 - Gráfico de Volumes nos Tanques de Querosene (Cenário 2)............ 94

Figura 50 - Gráfico de Volumes nos Tanques de Diesel (Cenário 2)................... 94

Figura 51 - Gráfico de Volumes nos Tanques de Diluente (Cenário 2). .............. 95

Figura 52 - Gráfico de Volumes nas Esferas de GLP (Cenário 2)........................ 95

Figura 53 - Gráfico de densidade nos tanques de diesel (Cenário 1)....................96

Figura 54 - Gráfico de enxofre nos tanques de diesel (Cenário 1).......................96 


\section{Lista de Tabelas}

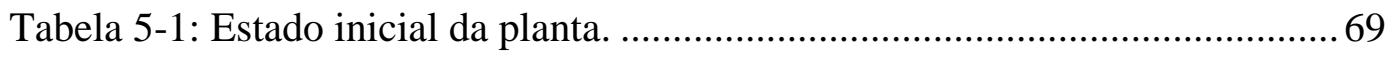

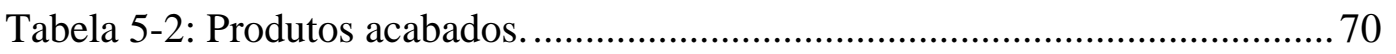

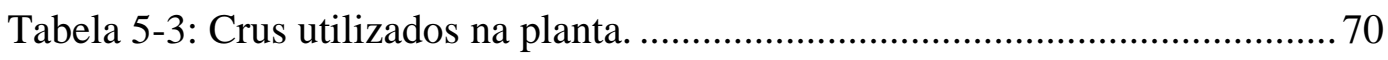

Tabela 5-4: Rendimentos de cada cru por campanha (fração volumétrica). ......... 70

Tabela 5-5: Propriedades nas correntes de saída por campanha. ........................... 70

Tabela 5-6: Desempenho geral da melhor solução encontrada para cada cenário.83 
O indivíduo que trabalha acerca-se continuamente do autor de todas as coisas, tomando na sua obra uma parte, de que depende também a dele. O criador começa, e a criatura acaba a criação de si própria.

Rui Barbosa, Oração aos Moços 